\title{
La evaluación funcional respiratoria en medicina ocupacional
}

\author{
Luis Torre-Bouscoulet $\bowtie$
}

Instituto Nacional de Enfermedades Respiratorias Ismael Cosío Villegas, Ciudad de México.

Trabajo recibido: 15-VII-2016; aceptado: 21-VII-2016

Los programas de vigilancia respiratoria en medicina ocupacional incluyen una radiografía de tórax y una espirometría simple. Aunque útiles, ambas son pruebas con una elevada tasa de falsos negativos; es decir, fallan en identificar procesos patológicos, especialmente en las etapas iniciales de las enfermedades. Lo anterior contrasta con el objetivo central de un programa de vigilancia laboral que es la identificación oportuna de anormalidades que sustenten un cambio en la actividad laboral o una intervención específica. La «identificación oportuna» hace referencia a la etapa preclínica en la que no existen síntomas y en la que evitar la exposición será una medida altamente eficiente para evitar la progresión del daño.

Las pruebas diagnósticas tienen un balance inverso entre sensibilidad y especificidad; cuando ganamos en uno, perdemos en otro. ${ }^{1}$ Como estrategia de salud pública, la implementación de medidas encaminadas para identificar oportunamente a los casos incidentes, es una medida costo-efectiva. La «búsqueda de casos», a diferencia del "escrutinio", aplica para población en riesgo -con elevada probabilidad preprueba-; por ejemplo, se buscarán casos de neumoconiosis en pacientes expuestos a polvos inorgánicos. El escrutinio -screening en inglés- hace referencia a la aplicación de una prueba diagnóstica que se realiza en una población independientemente del riesgo; por ejemplo, medir la glucosa en todos los adultos, independientemente de la edad, índice de masa corporal o antecedentes familiares de diabetes mellitus. ${ }^{1}$ Así, la evaluación funcional respiratoria en el medio ocupacional debería estar orientada a la búsqueda de casos mediante pruebas de elevada sensibilidad. Un sujeto con alta probabilidad preprueba que resulta positivo a una prueba diagnóstica de elevada sensibilidad, es improbable que no tenga la enfermedad en cuestión. Realizar pruebas de baja sensibilidad en individuos con alta probabilidad preprueba podría retrasar el diagnóstico por años hasta que la enfermedad esté tan avanzada que genere síntomas o anormalidades radiológicas. En esa condición, el programa de "vigilancia» habrá fallado. Esperar a que una enfermedad avance a tal grado que sea evidente en una prueba de baja sensibilidad -como la espirometría o la radiografía de tórax- tiene un costo muy elevado en términos de la salud de los trabajadores.

En el momento actual, la evaluación funcional respiratoria incluye un grupo de pruebas con diferentes grados de rendimiento diagnóstico. Aunque la espirometría es la mejor prueba disponible para evaluar la mecánica de la respiración, ${ }^{2}$ existen algunas estrategias que mejoran su rendimiento. La buena calidad técnica de la prueba es indispensable para hacer una correcta interpretación de la misma. De ahí la importancia de que la persona que lleva a cabo la espirometría esté lo suficientemente capacitada y se apegue a los lineamientos universales de calidad espirométrica. ${ }^{2}$ En México, existen cursos de certificación para realizar espirometrías que son avalados por el Instituto Nacional para la Salud y la Seguridad Ocupacional de los Estados Unidos de Norteamérica (NIOSH, por sus siglas en inglés). Además de la buena calidad técnica, otra estrategia que incrementa el rendimiento diagnóstico de la espirometría, es que se realice antes y después de administrar un broncodilatador. La respuesta que se tenga al broncodilatador puede hacer evidente anormalidades que no eran identificables con la espirometría basal. ${ }^{3}$ Estas características las tienen algunos pacientes con hiperreactividad bronquial. La selección apropiada de los valores de referencia es otra medida importante para la correcta interpretación de la prueba. Las ecuaciones 
disponibles tienen diferentes ajustes para una población dada. ${ }^{3}$ Nuestro grupo ha propuesto y evaluado diversas ecuaciones de referencia. ${ }^{4,5} \mathrm{~A}$ manera de resumen, la ecuación seleccionada debe ser aquella que mejor represente a la población en cuestión. ${ }^{5}$ Las ecuaciones de referencia son de utilidad para poder comparar los valores obtenidos de un individuo con aquéllos que se esperarían de una persona de sus mismas características. Esta comparación es inevitable en el análisis transversal de la función respiratoria.

La mejor comparación es cuando tenemos una espirometría previa. En este contexto, el sujeto es su propio control, su propia referencia. Así, la comparación no va a ser con un sujeto de sus mismas características, sino contra él mismo. Esa estrategia permitiría poner en evidencia cambios significativos en la función respiratoria que, si la comparación fuera contra una ecuación de referencia, podrían no haber sido identificados. ${ }^{6,7}$ Por lo tanto, cuando se tienen disponibles varias espirometrías de un mismo individuo, debemos incluir de manera rutinaria la comparación contra sus propios valores obtenidos en las espirometrías previas y no limitarnos a la comparación contra un valor predicho. Una disminución anual de $15 \%$ de la capacidad vital forzada o en el volumen espiratorio forzado en el primer segundo, es motivo suficiente para realizar estudios complementarios. Otra ventaja de la evaluación longitudinal es que se puede analizar si la tasa de pérdida de función respiratoria en un período dado de tiempo, es la esperada por el proceso natural de envejecimiento; o bien, si se encuentra acelerada por algún proceso patológico atribuible a la exposición laboral o a otros agentes como el consumo de tabaco.

La difusión pulmonar de monóxido de carbono (DLCO) es una prueba sensible de intercambio gaseoso. En las enfermedades que afectan el intersticio pulmonar, como las neumoconiosis, las anormalidades en el intercambio de gases suelen preceder a las anormalidades en la mecánica respiratoria. Por ello, es razonable que la vigilancia respiratoria se lleve a cabo mediante la prueba de DLCO; ésta identificará anormalidades de manera más sensible que la espirometría. La DLCO debería ser una prueba rutinaria en un programa de vigilancia respiratoria cuando la exposición se asocie a daño en el parénquima pulmonar. La estandarización vigente de la prueba de DLCO con la técnica de respiración única, ${ }^{8}$ ha hecho de ella una prueba reproducible y muy útil en la evaluación longitudinal de la función respiratoria. Los dispositivos de medición de la DLCO son ahora accesibles y de relativamente bajo costo. Además, el desarrollo tecnológico ha logrado que en la actualidad existan difusores portátiles, lo que impulsa su uso en los programas de vigilancia respiratoria y en la práctica clínica.

La prueba de caminata de 6 minutos nos permite cuantificar la distancia que caminando recorre un sujeto en un período de 6 minutos. ${ }^{9}$ En esta prueba el sujeto establece la velocidad a la que camina y es difícil discernir si el individuo realizó su mejor esfuerzo, lo que tiene implicaciones en el caso de los «simuladores». La mejor prueba para evaluar la capacidad aeróbica de un individuo es la prueba cardiopulmonar de ejercicio (PCPE).$^{10}$ Esta prueba nos permite saber el consumo de oxígeno de una persona en condiciones de ejercicio máximo. Además, nos permite analizar con detalle los factores que limitan la capacidad de ejercicio (limitación cardíaca, limitación respiratoria, limitación muscular, desacondicionamiento) y es la mejor prueba para analizar la incapacidad. Esta prueba tiene parámetros muy sensibles para evaluar el esfuerzo del sujeto, lo que elimina el posible efecto de los «simuladores».

Mi visión acerca de los programas de vigilancia respiratoria en medicina ocupacional incluye dos componentes. El primero, una evaluación funcional que dependerá del tipo de daño asociado a la exposición; y el segundo, una red de laboratorios de función pulmonar para llevar a cabo pruebas avanzadas de función respiratoria. En este modelo, si la exposición se asocia a asma, la evaluación funcional respiratoria deberá incluir a la espirometría con broncodilatador cada 6 o 12 meses. Algunos sujetos, además, necesitarán una evaluación de varios días de duración con un flujómetro, con el propósito de identificar variabilidad diurna anormal de la función respiratoria de acuerdo a los días u horas de exposición. Otros, serán remitidos a los laboratorios especializados, ya que requerirán pruebas de reto bronquial. Los laboratorios de función pulmonar que conformen la red, deberán seguir de manera estricta las recomendaciones internacionales para la ejecución de las pruebas y garantizar el control de calidad. En aquellas exposiciones que afectan directamente al parénquima pulmonar (por ejemplo, polvos inorgánicos o productos químicos asociados a enfisema), la evaluación deberá incluir a la espirometría con broncodilatador y una prueba de DLCO. Los casos seleccionados deberán ser referidos a la red de laboratorios, ya que podrían necesitar una prueba de caminata de 6 minutos o pruebas de ejercicio máximo.

\section{REFERENCIAS}

1. Gordis L. Assessing the validity and reliability of diagnostic and screening test. In: Epidemiology. 5th edition. ISBN 978-1-4557-3733-8. Canada: Elsevier Saunders; 2014.p. 3733-3738. 
2. Miller MR, Hankinson J, Brusasco V, et al.; ATS/ERS Task Force. Standardisation of spirometry. Eur Respir J 2005;26(2):319-338.

3. Pellegrino R, Viegi G, Brusasco V, et al. Interpretative strategies for lung function tests. Eur Respir J 2005;26(5):948-968.

4. Pérez-Padilla R, Valdivia G, Muiño A, et al. Spirometric reference values in 5 large Latin American cities for subjects aged 40 years or over. Arch Bronconeumol 2006;42(7):317-325.

5. Torre-Bouscoulet L, Pérez-Padilla R; Grupo de Trabajo del Estudio PLATINO en México. Adjustment of several spirometric reference equations to a population-based sample in Mexico. Salud Publica Mex 2006;48(6):466473.

6. Martínez-Briseño D, Fernández-Plata R, GochicoaRangel $\mathrm{L}$, et. al. Longitudinal lung function growth of Mexican children compared with international studies. PLoS One 2013;8(10):e77403. doi: 10.1371/journal. pone.0077403.

7. Skloot GS, Schechter CB, Herbert R, et al. Longitudinal assessment of spirometry in the World Trade Center medical monitoring program. Chest 2009;135(2):492498. doi: 10.1378/chest.08-1391.
8. Macintyre N, Crapo RO, Viegi G, et al. Standardisation of the single-breath determination of carbon monoxide uptake in the lung. Eur Respir J 2005; 26(4):720-735.

9. Gochicoa-Rangel L, Mora-Romero U, Guerrero-Zúñiga S, et al. Prueba de Caminata de 6 Minutos: Recomendaciones y procedimiento. Neumol Cir Torax 2015;74(2):127136.

10. Cid-Juárez S, Miguel-Reyes JL, Cortés-Télles A, Gochicoa-Rangel L, Silva-Cerón M, Torre-Bouscoulet L. Prueba cardiopulmonar de ejercicio. Recomendaciones y Procedimiento. Neumol Cir Torax 2015;74(3):207220.

\section{$\triangle$ Correspondencia:}

Dr. Luis Torre-Bouscoulet, Subdirector de Investigación Clínica, Instituto Nacional de Enfermedades Respiratorias Ismael Cosío Villegas. Calzada de Tlalpan Núm. 4502, colonia Sección XVI, 14080, Ciudad de México.

Tel (52) (55) 54871700; ext. 5160

Correo electrónico: luistorreb@gmial.com

El autor declara no tener conflicto de intereses. 